\title{
Dependence of nonlocal Gilbert damping on the ferromagnetic layer type in $\mathrm{FM} / \mathrm{Cu} / \mathrm{Pt}$ heterostructures
}

\author{
A. Ghosh, J.F. Sierra, S. Auffret, U. Ebels ${ }^{1}$ and W.E. Bailey ${ }^{2}$ \\ 1) SPINTEC, UMR(8191) CEA / CNRS / UJF / Grenoble INP ; INAC, \\ 17 rue des Martyrs, 38054 Grenoble Cedex, France \\ 2) Dept. of Applied Physics \& Applied Mathematics, Columbia University, \\ New York NY 1002\%, USA
}

(Dated: 7 September 2021)

We have measured the size effect in nonlocal Gilbert relaxation rate in $\mathrm{FM}\left(\mathrm{t}_{F M}\right) /$ $\mathrm{Cu}(5 \mathrm{~nm})[/ \mathrm{Pt}(2 \mathrm{~nm})] / \mathrm{Al}(2 \mathrm{~nm})$ heterostructures, $\mathrm{FM}=\left\{\mathrm{Ni}_{81} \mathrm{Fe}_{19}, \mathrm{Co}_{60} \mathrm{Fe}_{20} \mathrm{~B}_{20}\right.$, pure Co\}. Common behavior is observed for three FM layers, where the additional relaxation obeys both a strict inverse power law dependence $\Delta G=K t^{n}, n=-1.04 \pm$ 0.06 and a similar magnitude $K=224 \pm 40 \mathrm{Mhz} \cdot \mathrm{nm}$. As the tested FM layers span an order of magnitude in spin diffusion length $\lambda_{S D L}$, the results are in support of spin diffusion, rather than nonlocal resistivity, as the origin of the effect. 
The primary materials parameter which describes the temporal response of magnetization $\mathbf{M}$ to applied fields $\mathbf{H}$ is the Gilbert damping parameter $\alpha$, or relaxation rate $G=|\gamma| M_{s} \alpha$. Understanding of the Gilbert relaxation, particularly in structures of reduced dimension, is an essential question for optimizing the high speed / Ghz response of nanoscale magnetic devices.

Experiments over the last decade have established that the Gilbert relaxation of ferromagnetic ultrathin films exhibits a size effect, some component of which is nonlocal. Both $\alpha\left(t_{F M}\right)=\alpha_{0}+\alpha^{\prime}\left(t_{F M}\right)$ and $G\left(t_{F M}\right)=G_{0}+G^{\prime}\left(t_{F M}\right)$ increase severalfold with decreasing FM film thickness $t_{F M}$, from near-bulk values $\alpha_{0}, G_{0}$ for $t_{F M} \gtrsim 20 \mathrm{~nm}$. Moreover, the damping size effect can have a nonlocal contribution responsive to layers or scattering centers removed, through a nonmagnetic (NM) layer, from the precessing FM. Contributed Gilbert relaxation has been seen from other FM layers $\stackrel{1}{\text { as }}$ well as from heavy-element scattering layers such as $\mathrm{Pt} \stackrel{2}{2}$

The nonlocal damping size effect is strongly reminiscent of the electrical resistivity in ferromagnetic ultrathin films. Electrical resistivity $\rho$ is size-dependent by a similar factor over a similar range of $t_{F M}$; the resistivity $\rho\left(t_{F M}\right)$ is similarly nonlocal, dependent upon layers not in direct contact. $\underline{\underline{3}} \underline{\underline{5}}$. It is prima facie plausible that the nonlocal damping and nonlocal electrical resistivity share a common origin in momentum scattering (with relaxation time $\left.\tau_{M}\right)$ by overlayers. If the nonlocal damping arises from nonlocal scattering $\tau_{M}^{-1}$, however, there should be a marked dependence upon FM layer type. Damping in materials with short spin diffusion length $\lambda_{S D L}$ is thought to be proportional to $\tau_{M}^{-1}$ (ref. $\underline{6}$ ); the claim for "resistivity-like" damping has been made explicitly for $\mathrm{Ni}_{81} \mathrm{Fe}_{19}$ by Ingvarsson ${ }^{7}$ et al. For FM with a long $\lambda_{S D L}$, on the other hand, relaxation $G$ is either nearly constant with temperature or "conductivity-like," scaling as $\tau_{M}$.

Interpretation of the nonlocal damping size effect has centered instead on a spin current model ${ }^{\underline{8}}$ advanced by Tserkovnyak et $\mathrm{al}^{\underline{9}}$. An explicit prediction of this model is that the magnitude of the nonlocal Gilbert relaxation rate $\Delta G$ is only weakly dependent upon the FM layer type. The effect has been calculated 10 as

$$
\Delta G=|\gamma|^{2} \hbar / 4 \pi\left(g_{\text {eff }}^{\uparrow \downarrow} / S\right) t_{F M}^{-1}
$$

, where the effective spin mixing conductance $g_{\text {eff }}^{\uparrow \downarrow} / S$ is given in units of channels per area. Ab-initio calculations predict a very weak materials dependence for the interfacial parameters 
$g^{\uparrow \downarrow} / S$, with $\pm 10 \%$ difference in systems as different as $\mathrm{Fe} / \mathrm{Au}$ and $\mathrm{Co} / \mathrm{Cu}$, and negligible dependence on interfacial mixing: $\underline{\underline{11}}$

Individual measurements exist of the spin mixing conductance, through the damping, in $\mathrm{FM}$ systems $\mathrm{Ni}_{81} \mathrm{Fe}_{19} \stackrel{12}{\underline{2}}, \mathrm{Co} \underline{\underline{13}}$, and $\mathrm{CoFeB} \underline{\underline{14}}$. However, these experiments do not share a common methodology, which makes a numerical comparison of the results problematic, especially given that Gilbert damping estimates are to some extent model-dependent $\frac{15}{\underline{15}}$. In our experiments, we have taken care to isolate the nonlocal damping contribution due to $\mathrm{Pt}$ overlayers only, controlling for growth effects, interfacial intermixing, and inhomogeneous losses. The only variable in our comparison of nonlocal damping $\Delta G\left(t_{F M}\right)$, to the extent possible, has been the identity of the FM layer.

Gilbert damping $\alpha$ has been measured through ferromagnetic resonance (FMR) from $\omega / 2 \pi=2-24 \mathrm{Ghz}$ using a broadband coplanar waveguide (CPW) with broad center conductor width $w=400 \mu m$, using field modulation and lock-in detection of the transmitted signal to enhance sensitivity. The Gilbert damping has been separated from inhomogeneous broadening in the films measured using the well-known relation $\Delta H_{p p}(\omega)=\Delta H_{0}+(2 / \sqrt{3}) \alpha \omega /|\gamma|$. We have fit spectra to Lorenzian derivatives with Dysonian components at each frequency, for each film, to extract the linewidth $\Delta H_{p p}$ and resonance field $H_{r e s} ; \alpha$ has been extracted using linear fits to $\Delta H(\omega)$.

For the films, six series of heterostructures were deposited of the form $\mathrm{Si} / \mathrm{SiO}_{2} /$ $\mathrm{X} / \mathrm{FM}\left(t_{F M}\right) / \mathrm{Cu}(3 \mathrm{~nm})[/ \mathbf{P t}(\mathbf{3 n m})] / \mathrm{Al}(3 \mathrm{~nm}), \quad \mathrm{FM}=\left\{\mathrm{Ni}_{81} \mathrm{Fe}_{19}\right.$ ("Py"), $\mathrm{Co}_{60} \mathrm{Fe}_{20} \mathrm{~B}_{20}$ ("CoFeB"), pure Co , and $t_{F M}=2.5,3.5,6.0,10.0,17.5,30.0 \mathrm{~nm}$, for 36 heterostructures included in the study. For each ferromagnetic layer type $F M$, one thickness series $t_{F M}$ was deposited with the Pt overlayer and one thickness series $t_{F M}$ was deposited without the Pt overlayer. This makes it possible to record the additional damping $\Delta \alpha\left(t_{F M}\right)$ introduced by the Pt overlayer alone, independent of size effects present in the FM/Cu layers deposited below. In the case of pure $\mathrm{Co}$, a $\mathrm{X}=\mathrm{Ta}(5 \mathrm{~nm}) / \mathrm{Cu}(5 \mathrm{~nm})$ underlayer was necessary to stabilize low-linewidth films, otherwise, depositions were carried out directly upon the in-situ ion-cleaned substrate.

Field-for-resonance data are presented in Figure 1. The main panel shows $\omega\left(H_{B}^{\|}\right)$data for $\mathrm{Ni}_{81} \mathrm{Fe}_{19}\left(t_{F M}\right)$. Note that there is a size effect in $\omega\left(H_{B}^{\|}\right)$: the thinner films have a substantially lower resonance frequency. For $t_{F M}=2.5 \mathrm{~nm}$, the resonance frequency is depressed by $\sim 5 \mathrm{Ghz}$ from $\sim 20 \mathrm{Ghz}$ resonance $H_{B} \simeq 4 \mathrm{kOe}$. The behavior is fitted through 
the Kittel relation (lines) $\omega\left(H_{B}^{\|}\right)=|\gamma| \sqrt{\left(H_{B}^{\|}+H_{K}\right)\left(4 \pi M_{s}^{e f f}+H_{B}^{\|}+H_{K}\right)}$, and the inset shows a summary of extracted $4 \pi M_{s}^{e f f}\left(t_{F M}\right)$ data for the three different FM layers. Samples with (open symbols) and without (closed symbols) Pt overlayers show negligible differences. Linear fits according to $4 \pi M_{s}^{e f f}\left(t_{F M}\right)=4 \pi M_{s}-\left(2 K_{s} / M_{s}\right) t_{F M}^{-1}$ allow the extraction of bulk magnetization $4 \pi M_{s}$ and surface anisotropy $K_{s}$; we find $4 \pi M_{s}^{P y}=10.7 \mathrm{kG}, 4 \pi M_{s}^{C o F e B}=$ $11.8 \mathrm{kG}, 4 \pi M_{s}^{C o}=18.3 \mathrm{kG}$, and $K_{s}^{P y}=0.69 \mathrm{erg} / \mathrm{cm}^{2}, K_{s}^{C o F e B}=0.69 \mathrm{erg} / \mathrm{cm}^{2}, K_{s}^{C o}=$ $1.04 \mathrm{erg} / \mathrm{cm}^{2}$. The value of $g_{L} / 2=|\gamma| /(e / m c),|\gamma|=2 \pi \cdot(2.799 \mathrm{Mhz} / \mathrm{Oe}) \cdot\left(g_{L} / 2\right)$ is found from the Kittel fits subject to this choice, yielding $g_{L}^{P y}=2.09, g_{L}^{C o F e B}=2.07, g_{L}^{C o}=2.15$. The $4 \pi M_{s}$ and $g_{L}$ values, taken to be size-independent, are in good agreement with bulk values.

FMR linewidth as a function of frequency $\Delta H_{p p}(\omega)$ is plotted in Figure 2, The data for Py show a near-proportionality, with negligble inhomogeneous component $\Delta H_{0} \leq 4$ Oe even for the the thinnest layers, facilitating the extraction of intrinsic damping parameter $\alpha$. The size effect in in $\alpha\left(t_{F M}\right)$ accounts for an increase by a factor of $\sim 3$, from $\alpha_{0}^{P y}=$ $0.0067\left(G_{0}^{P y}=105 \mathrm{Mhz}\right)$ for the thickest films $\left(t_{F M}=30.0 \mathrm{~nm}\right)$ to $\alpha=0.021$ for the thinnest films $\left(t_{F M}=2.5 \mathrm{~nm}\right)$. The inset shows the line shapes for films with and without Pt, illustrating the broadening without significant frequency shift or significant change in peak asymmetry.

A similar analysis has been carried through for $\mathrm{CoFeB}$ and $\mathrm{Co}$ (not pictured). Larger inhomogeneous linewidths are observed for pure Co, but homogeneous linewidth still exceeds inhomogeneous linewidth by a factor of three over the frequency range studied, and inhomogeneous linewidths agree within experimental error for the thinnest films with and without Pt overlayers. We extract for these films $\alpha_{0}^{C o F e B}=0.0065\left(G_{0}^{C o F e B}=111 \mathrm{Mhz}\right)$ and $\alpha_{0}^{C o}=0.0085\left(G_{0}^{C o}=234 \mathrm{Mhz}\right)$. The latter value is in very good agreement with the average of easy- and hard-axis values for epitaxial FCC Co films measured up to 90 Ghz, $G_{0}^{C o}=225 \mathrm{Mhz} \cdot \underline{16}$

We isolate the effect of Pt overlayers on the damping size effect in Figure 3, Values of $\alpha$ have been fitted for each deposited heterostructure: each FM type, at each $t_{F M}$, for films with and without Pt overlayers. We take the difference $\Delta \alpha\left(t_{F M}\right)$ for identical $\mathrm{FM}\left(t_{F M}\right) / \mathrm{Cu}(5 \mathrm{~nm}) / \mathrm{Al}(3 \mathrm{~nm})$ depositions with and without the insertion of $\mathrm{Pt}(3 \mathrm{~nm})$ after the $\mathrm{Cu}$ deposition. Data, as shown on the logarithmic plot in the main panel, are found 
to obey a power law $\Delta \alpha\left(t_{F M}\right)=K t^{n}$, with $n=-1.04 \pm 0.06$. This is excellent agreement with an inverse thickness dependence $\Delta \alpha\left(t_{F M}\right)=K_{F M} / t_{F M}$, where the prefactor clearly depends on the FM layer, highest for Py and lowest for Co. Note that efforts to extract $\Delta \alpha\left(t_{F M}\right)=K t^{n}$ without the $\mathrm{FM}\left(t_{F M}\right) / \mathrm{Cu}$ baselines would meet with significant errors; numerical fits to $\alpha\left(t_{F M}\right)=K t_{F M}{ }^{n}$ for the $\mathrm{FM}\left(t_{F M}\right) / \mathrm{Cu} / \mathrm{Pt}$ structures yield exponents $n \simeq 1.4$.

Expressing now the additional Gilbert relaxation as $\Delta G\left(t_{F M}\right)=|\gamma| M_{s} \Delta \alpha\left(t_{F M}\right)=$ $\left|\gamma^{F M}\right| M_{s}^{F M} K_{F M} / t_{F M}$, we plot $\Delta G \cdot t_{F M}$ in Figure 4. We find $\Delta G \cdot t_{P y}=192 \pm 40 \mathrm{Mhz}$, $\Delta G \cdot t_{C o F e B}=265 \pm 40 \mathrm{Mhz}$, and $\Delta G \cdot t_{C o}=216 \pm 40 \mathrm{Mhz}$. The similarity of values for $\Delta G \cdot t_{F M}$ is in good agreement with predictions of the spin pumping model in Equation 1, given that interfacial spin mixing parameters are nearly equal in different systems.

The similarity of the $\Delta G \cdot t_{F M}$ values for the different FM layers is, however, at odds with expectations from the "resistivity-like" mechanism. In Figure 4 , inset, we show the dependence of $\Delta G \cdot t_{F M}$ upon the tabulated $\lambda_{S D L}$ of these layers from Ref17. It can be seen that $\lambda_{S D L}^{C o}$ is roughly an order of magnitude longer than it is for the other two FM layers, $\mathrm{Py}$ and $\mathrm{CoFeB}$, but the contribution of $\mathrm{Pt}$ overlayers to damping is very close to their average. Since under the resistivity mechanism, only $\mathrm{Py}$ and $\mathrm{CoFeB}$ should be susceptible to a resistivity contribution in $\Delta \alpha\left(t_{F M}\right)$, the results imply that the contribution of $\mathrm{Pt}$ to the nonlocal damping size effect has a separate origin.

Finally, we compare the magnitude of the nonlocal damping size effect with that predicted by the spin pumping model in Ref. $\underline{\underline{10}}$. According to $\Delta G \cdot t_{F M}=|\gamma|^{2} \hbar / 4 \pi=$ 25.69 Mhz $\cdot \mathrm{nm}^{3}\left(g_{L} / 2\right)^{2}\left(g_{\text {eff }}^{\uparrow \downarrow} / S\right)$, our experimental $\Delta G \cdot t_{F M}$ and $g_{L}$ data yield effective spin mixing conductances $g_{\text {eff }}^{\uparrow \downarrow} / S[\mathrm{Py} / \mathrm{Cu} / \mathrm{Pt}]=6.8 \mathrm{~nm}^{-2}, g_{\text {eff }}^{\uparrow \downarrow} / S[\mathrm{Co} / \mathrm{Cu} / \mathrm{Pt}]=7.3 \mathrm{~nm}^{-2}$, and $g_{\text {eff }}^{\uparrow \downarrow} / S[\mathrm{CoFeB} / \mathrm{Cu} / \mathrm{Pt}]=9.6 \mathrm{~nm}^{-2}$. The Sharvin-corrected form, in the realistic limit of $\lambda_{S D L}^{N} \gg t_{N} \underline{\underline{11}}$ is $\left(g_{\text {eff }}^{\uparrow \downarrow} / S\right)^{-1}=\left(g_{F / N}^{\uparrow \downarrow} / S\right)^{-1}-\frac{1}{2}\left(g_{N, S}^{\uparrow \downarrow} / S\right)^{-1}+2 e^{2} h^{-1} \rho t_{N}+\left(\tilde{g}_{N_{1} / N_{2}}^{\uparrow \downarrow} / S\right)^{-1}$. Using conductances $14.1 \mathrm{~nm}^{-2}(\mathrm{Co} / \mathrm{Cu}), 15.0 \mathrm{~nm}^{-2}(\mathrm{Cu}), 211 \mathrm{~nm}^{-2}$ (bulk $\left.\rho_{C u}, t_{N}=3 \mathrm{~nm}\right), 35$ $\mathrm{nm}^{-2}(\mathrm{Cu} / \mathrm{Pt})$ would predict a theoretical $g_{\text {eff,th. }}^{\uparrow \uparrow} / S[\mathrm{Co} / \mathrm{Cu} / \mathrm{Pt}]=14.1 \mathrm{~nm}^{-2}$. Reconciling theory and experiment would require an order of magnitude larger $\rho_{C u} \simeq 20 \mu \Omega \cdot \mathrm{cm}$, likely not physical.

To summarize, a common methodology, controlling for damping size effects and intermixing in single films, has allowed us to compare the nonlocal damping size effect in different FM layers. We observe, for $\mathrm{Cu} / \mathrm{Pt}$ overlayers, the same power law in thickness $t^{-1.04 \pm 0.06}$, 
the same materials independence, but roughly half the magnitude that predicted by the spin pumping theory of Tserkovnyak ${ }^{10}$. The rough independence on FM spin diffusion length, shown here for the first time, argues against a resistivity-based interpretation for the effect.

We would like to acknowledge the US NSF-ECCS-0925829, the Bourse Accueil Pro n 2715 of the Rhône-Alpes Region, the French National Research Agency (ANR) Grant ANR09-NANO-037, and the FP7-People-2009-IEF program no 252067.

\section{REFERENCES}

${ }^{1}$ R. Urban, G. Woltersdorf, and B. Heinrich, "Gilbert damping in single and multilayer ultrathin films: role of interfaces in nonlocal spin dynamics," Physical Review Letters 87, 217204-7 (2001).

${ }^{2}$ S. Mizukami, Y. Ando, and T. Miyazaki, "Effect of spin diffusion on Gilbert damping for a very thin permalloy layer in Cu/permalloy/Cu/Pt films," Phys. Rev. B 66, 104413 (2002). ${ }^{3}$ B. Dieny, J. Nozieres, V. Speriosu, B. Gurney, and D. Wilhoit, "Change in conductance is the fundamental measure of spin-valve magnetoresistance," Applied Physics Letters 61, 2111-3 (1992).

${ }^{4}$ W. H. Butler, X. G. Zhang, D. M. C. Nicholson, T. C. Schulthess, and J. M. MacLaren, "Giant magnetoresistance from an electron waveguide effect in cobalt-copper multilayers," Physical Review Letters 76, 3216-19 (1996).

${ }^{5}$ W. E. Bailey, S. X. Wang, and E. Y. Tsymbal, "Electronic scattering from $\mathrm{Co} / \mathrm{Cu}$ interfaces: In situ measurement and comparison with theory," Phys. Rev. B 61, 1330-1335 (2000).

${ }^{6} \mathrm{~V}$. Kamberský, "On the landau-lifshitz relaxation in ferromagnetic metals," Canadian Journal of Physics 48, 2906 (1970).

${ }^{7}$ S. Ingvarsson, L. Ritchie, X. Liu, G. Xiao, J. Slonczewski, P. Trouilloud, and R. Koch, "Role of electron scattering in the magnetization relaxation of thin $\mathrm{Ni}_{81} \mathrm{Fe}_{19}$ films," Phys. Rev, B, Condens, Matter Mater. Phys. (USA) 66, 214416 - 1 (2002/12/01),

${ }^{8}$ R. Silsbee, A. Janossy, and P. Monod, "Coupling between ferromagnetic and conductionspin-resonance modes at a ferromagnetic normal-metal interface," Physical Review B (Condensed Matter) 19, 4382 - 99 (1979).

${ }^{9}$ Y. Tserkovnyak, A. Brataas, and G. E. W. Bauer, "Enhanced Gilbert damping in thin 
ferromagnetic films," Phys. Rev. Lett. 88, 117601 (2002).

${ }^{10} \mathrm{Y}$. Tserkovnyak, A. Brataas, G. Bauer, and B. Halperin, "Nonlocal magnetization dynamics in ferromagnetic heterostructures," Reviews in Modern Physics 77, 1375 - 421 (2005).

${ }^{11}$ M. Zwierzycki, Y. Tserkovnyak, P. J. Kelly, A. Brataas, and G. E. W. Bauer, "Firstprinciples study of magnetization relaxation enhancement and spin transfer in thin magnetic films," Phys. Rev. B 71, 064420 (2005).

${ }^{12}$ S. Mizukami, Y. Ando, and T. Miyazaki, "Magnetic relaxation of normal-metal NM/NiFe/NM films," Journal of Magnetism and Magnetic Materials 239, 42 - 4 (2002); "The study on ferromagnetic resonance linewidth for NM/ 80NiFe/ NM, $(\mathrm{NM}=\mathrm{Cu}$, Ta, Pd and Pt) films," Japanese Journal of Applied Physics, Part 1 (Regular Papers, Short Notes and Review Papers) 40, 580 - 5 (2001).

${ }^{13}$ J. Beaujour, W. Chen, A. Kent, and J. Sun, "Ferromagnetic resonance study of polycrystalline cobalt ultrathin films," Journal of Applied Physics 99, 08N503 (2006); J.-M. Beaujour, J. Lee, A. Kent, K. Krycka, and C.-C. Kao, "Magnetization damping in ultrathin polycrystalline co films: evidence for nonlocal effects," Physical Review B (Condensed Matter and Materials Physics) 74, 214405-1 (2006).

${ }^{14}$ H. Lee, L. Wen, M. Pathak, P. Janssen, P. LeClair, C. Alexander, C. Mewes, and T. Mewes, "Spin pumping in $\mathrm{Co}_{56} \mathrm{Fe}_{24} \mathrm{~B}_{20}$ multilayer systems," Journal of Physics D: Applied Physics 41, 215001 (5 pp.) - (2008).

${ }^{15}$ R. McMichael and P. Krivosik, "Classical model of extrinsic ferromagnetic resonance linewidth in ultrathin films," IEEE Transactions on Magnetics 40, 2 - 11 (2004).

16 "Gilbert damping and g-factor in $\mathrm{Fe}_{x} \mathrm{Co}_{1-x}$ alloy films," Solid State Communications 93, $965-968$ (1995).

${ }^{17}$ J. Bass and J. Pratt, W.P., "Spin-diffusion lengths in metals and alloys, and spin-flipping at metal/metal interfaces: an experimentalist's critical review," Journal of Physics: Condensed Matter 19, 41 pp. - (2007); C. Ahn, K.-H. Shin, and W. Pratt, "Magnetotransport properties of $\mathrm{CoFeB}$ and $\mathrm{Co} / \mathrm{Ru}$ interfaces in the current-perpendicular-to-plane geometry," Applied Physics Letters 92, 102509 - 1 (2008). 


\section{FIGURES}

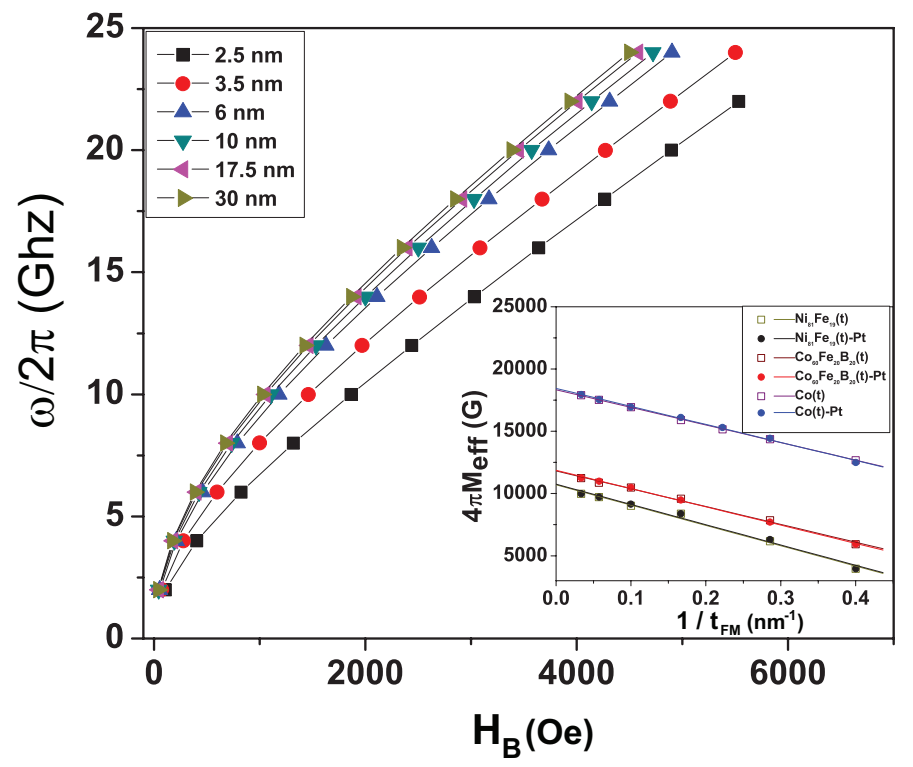

FIG. 1. Fields for resonance $\omega\left(H_{B}\right)$ for in-plane FMR, $\mathrm{FM}=\mathrm{Ni}_{81} \mathrm{Fe}_{19}, 2.5 \mathrm{~nm} \leq t_{F M} \leq 30.0 \mathrm{~nm}$; solid lines are Kittel fits. Inset: $4 \pi \mathrm{M}_{s}^{e f f}$ for all three $\mathrm{FM} / \mathrm{Cu}$, with and without Pt overlayers.

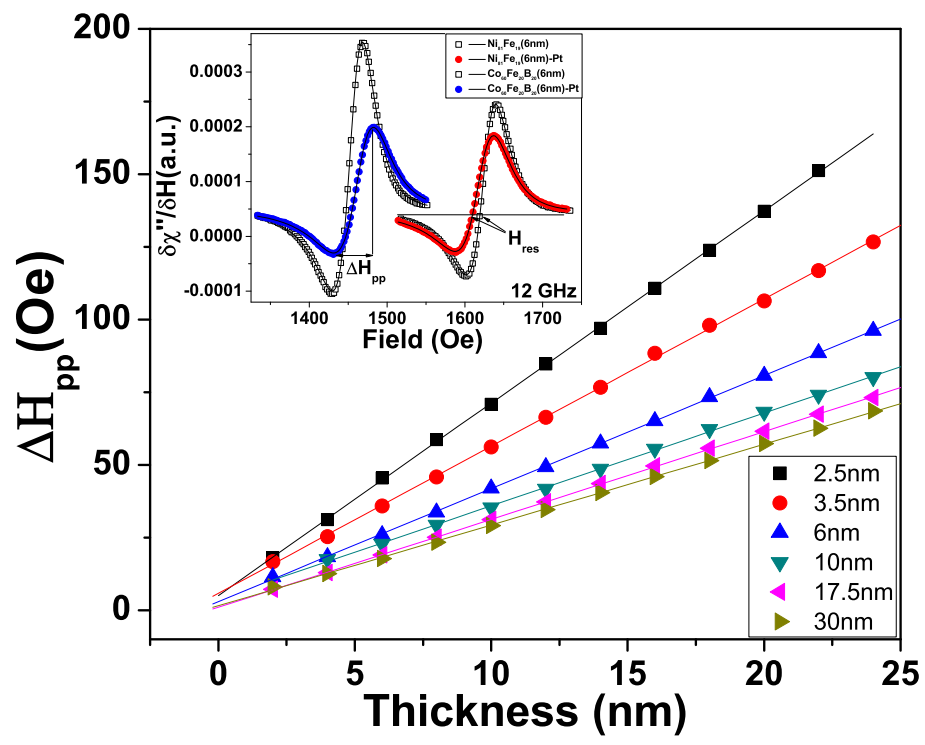

FIG. 2. Frequency-dependent peak-to-peak FMR linewidth $\Delta H_{p p}(\omega)$ for $\mathrm{FM}=\mathrm{Ni}_{81} \mathrm{Fe}_{19}, t_{F M}$ as noted, films with Pt overlayers. Inset: lineshapes and fits for films with and without Pt, $\mathrm{FM}=\mathrm{Ni}_{81} \mathrm{Fe}_{19}, \mathrm{CoFeB}$. 


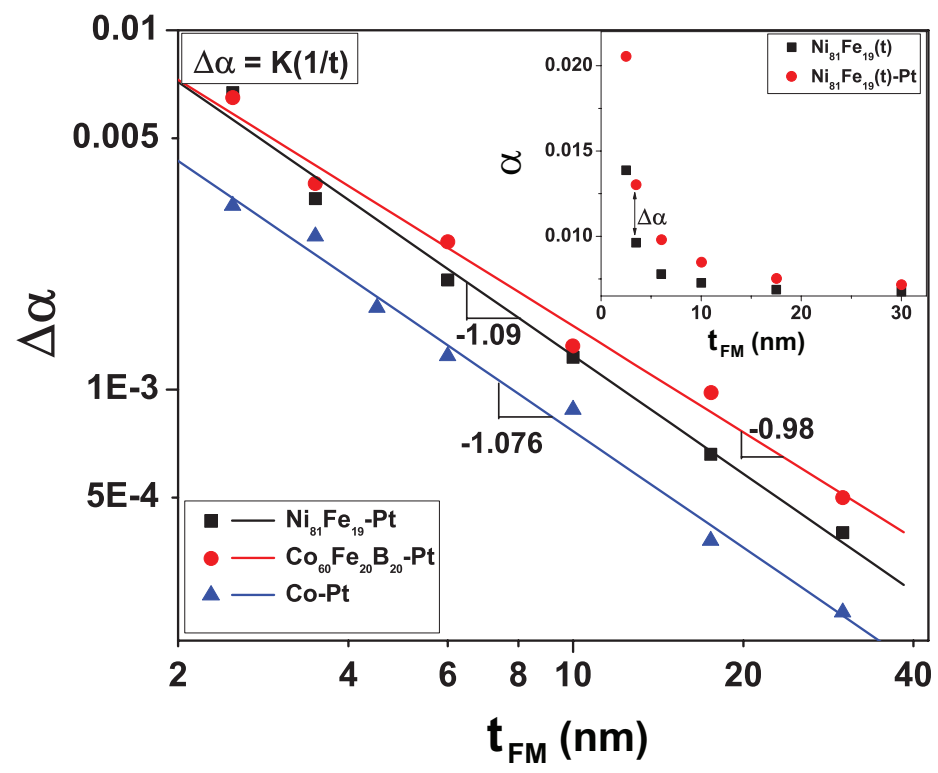

FIG. 3. Inset: $\alpha_{\text {no Pt }}\left(t_{F M}\right)$ and $\alpha_{\mathrm{Pt}}$ for Py, after linear fits to data in Figure 2, Main panel: $\Delta \alpha\left(t_{F M}\right)=\alpha_{\mathrm{Pt}}\left(t_{F M}\right)-\alpha_{\text {no Pt }}\left(t_{F M}\right)$ for $\mathrm{Py}, \mathrm{CoFeB}$, and Co. The slopes express the power law exponent $n=-1.04 \pm 0.06$.

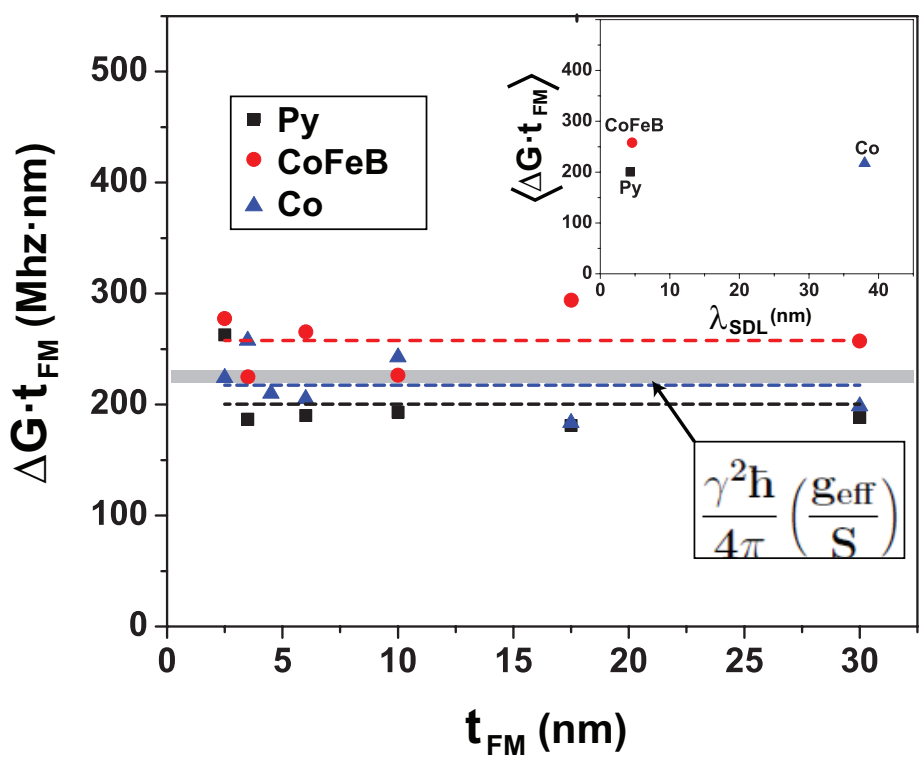

FIG. 4. The additional nonlocal relaxation due to Pt overlayers, expressed as a Gilbert relaxation rate - thickness product $\Delta G \cdot t_{F M}$ for $\mathrm{Py}, \mathrm{CoFeB}$, and Co. Inset: dependence of $\Delta G \cdot t_{F M}$ on spin diffusion length $\lambda_{S D L}$ as tabulated in $\frac{17}{}$. 